\title{
Faillure Analysis of Industrial Discharge Hopper Pipe
}

\author{
S. Rajole ${ }^{1}$, P. R. Sondar², S. Hiremath ${ }^{3}$, K. S Ravishankar4. \\ ${ }^{1}$ Faculty of Department of Mechanical Engineering, School of Engineering, Central University of Karnataka, Kalburgi 585367, India \\ ${ }^{2}$ Research Scholar of Department of Metallurgical and Materials Engineering, National Institute of Technology Karnataka, Surathkal 575025, India \\ ${ }^{3}$ Faculty of Department of Mechatronics Engineering, Manipal Institute of Technology, Manipal 576104, India \\ ${ }^{4}$ Faculty of Department of Metallurgical and Materials Engineering, National Institute of Technology Karnataka, Surathkal 575025, India
}

\begin{abstract}
This work presents the detailed investigation and root cause analysis of catastrophic failure of an industrial discharge hopper pipe. The hopper pipe investigated in the present work is made of austenitic stainless steel, 316 grade, a commonly used grade for valves, pipes and heat exchanger tubes. The pipe fractured in transverse direction, leading to the failure of discharge pipe. Visual inspection, dye penetrant test, chemical analysis, microstructural analysis, and fractography analysis were carried out on the failed part and it was concluded that stress corrosion cracking led to the failure of hopper pipe. The pipe constantly being under the exposure of industrial water and the presence of chlorine content in it made it susceptible for stress corrosion cracking. Transgranular fracture was clearly observed in the microstructure of failed sample and spot EDX at the fracture location confirmed the presence of chlorine content. Further, fractography showed striations indicating fatigue loading. The work concluded that stress corrosion cracking as the root cause of failure.
\end{abstract}

\section{ARTICLE HISTORY}

Revised: $13^{\text {rd }}$ November 2020

Accepted: $16^{\text {th }}$ November 2020

\section{KEYWORDS}

Austenitic Stainless Steel, Transgranular Fracture,

Stress Corrosion Cracking

\section{INTRODUCTION}

Most of the oil and natural gas industries use austenitic stainless steels as their piping material, as they have good corrosion resistance and mechanical strength [1], [2]. Even though austenitic stainless steel is corrosion resistant and has superior mechanical properties, operational and maintenance errors may cause premature failure [3]. Several failures of the industrial pipes, that have been reported in the past are mostly due to Stress Corrosion Cracking (SCC), sensitization and hydrogen embrittlement [4]-[6]. Stress Corrosion Cracking is a delayed failure where corrosion environments such as condensed water, ammonia solutions, moist air, solutions containing chlorides and nitrides play a major role. These corrosion products in combination with the stresses in the material due to service conditions lead to failure of the material. Austenitic stainless steels are also susceptible to Sensitization that occurs at particular temperature ranging from 480 to $815^{\circ} \mathrm{C}$, as chromium depletion occurs at this temperature leading to failure of material to resist corrosion. Hydrogen embrittlement occurs when the material is subjected to hydrogen atmosphere, and the hydrogen atom can enter through the grain boundaries. This weakens the atomic bond and surface energy, resulting in catastrophic failure of material. Even though, stainless steels possess good resistance against the hydrogen embrittlement, it has been reported that certain grade of stainless steels, in particular, SS 300 series suffer hydrogen embrittlement [7]. In one of the reports it was stated that interaction of carbonate/bicarbonate solution having high $\mathrm{pH}$ with the outer surface of pipe along with tensile stresses caused stress corrosion cracking leading to the failure of gas transmission steel pipeline after 40 years of installation [8]. A similar study reported, carbonates and bicarbonates present in soil along with hoop stresses in the pipe cau sed sudden propagation of longitudinal cracks at the outer surface of the crack, causing stress corrosion cracking, leading to blow out of oil and natural gas transmission pipeline [9]. Chloride stress corrosion cracking was observed in another report, involving precipitation hardening stainless steel impeller of centrifugal compressor, used in gas lifting operation. Since, changing the material was expensive and changing the external environment was highly impossible, autocatalytic nickelphosphorous coating was preferred to avoid such kind of failures as the coating produces hard layer with uniform thickness even on sharp edges of the impeller [10]. A similar study showed, failure of stainless steel pipe in a Methyl Methacrylate plant as a result of stress corrosion cracking. Stress corrosion cracking occurred due to presence of concentrated chloride that remained inside the pipe. Stress corrosion initiated from the corrosion pits, and the residual stresses originated from welding attributed to propagation of crack causing failure of pipe [11]. Another study, involving failure of downhole pressure memory gauge cover, made of AISI 316 stainless steel, reported stress corrosion cracking as the root cause of failure. Corrosion pits acted as the initiation for the SCC and the external pressure along with aggressive medium of sour gas well, having chloride content in it helped in propagation of cracks. It was suggested to replace the 316 stainless steel by either 17-4 PH or Nitronic 50 stainless steel as they have high resistance for pitting corrosion [12]. Yet another study reported failure of electrohydraulic oil pipe as a result combination of chloride pitting corrosion and stress corrosion cracking in the humid environment [13]. In another study, critical failure of recycle oil pipe used in decomposition of heavy oil was reported. It was concluded that, micro fissures formed at the pipe because of 
hydrogen attack. As the internal pressure built up and pipe was not able to endure the internal pressure because of fissure, the chemical substances present in it leaked out and caused explosion of the pipe [14].

Present investigation deals with failure of hopper pipe of oil and natural gas industry. Detailed investigation has been carried out involving Visual inspection, Dye Penetration Test, Microstructural Study and Fractography.

\section{MATERIALS AND METHODS}

The hopper pipe was made of 316 grade austenitic stainless steel, a commonly used stainless steel grade for valves, pipes and heat exchanger tubes. Considering the increased corrosion resistance 316 grade is largely used in chemical processing and storage equipment. To confirm the composition of the hopper tube, EDX was carried out on hopper tube sample. As shown in Table 1, composition of hopper pipe sample agrees well with 316 grade stainless steel. Further, Figure 1 shows the microstructure of the as received failed sample, highlighting typical austenite grains, confirming austenitic stainless steel grade.

Standard protocol of failure analysis was followed in analyzing the failure involving visual inspection, non destructive testing, microstructural analysis and fractography. Zeiss optical microscope was used for obtaining low magnification images whereas JEOL Scanning Electron Microscope (SEM) with secondary electron mode was used for obtaining high magnification images and Energy dispersion spectrometer (EDX) in combination with SEM was used for analyzing the chemical composition on the as received material and on fracture surfaces.

Table. 1 Nominal Chemical composition (in wt.\%) of grade 316 stainless steel in comparison with hopper pipe material

\begin{tabular}{cccccccc}
\hline & $\mathbf{C}$ & $\mathbf{M n}$ & $\mathbf{S i}$ & $\mathbf{N i}$ & $\mathbf{C r}$ & $\mathbf{M o}$ & $\mathbf{F e}$ \\
\hline 316 Grade & $\begin{array}{l}0.08 \\
\text { max. }\end{array}$ & $\begin{array}{l}2.0 \\
\text { max. }\end{array}$ & $\begin{array}{l}0.75 \\
\text { max. }\end{array}$ & $10.0-14.0$ & $16.0-18.0$ & $2.0-3.0$ & Bal. \\
$\begin{array}{c}\text { Hopper } \\
\text { pipe }\end{array}$ & 0.08 & 2.10 & 0.225 & 10.2 & 17.8 & 1.96 & Bal. \\
\hline
\end{tabular}

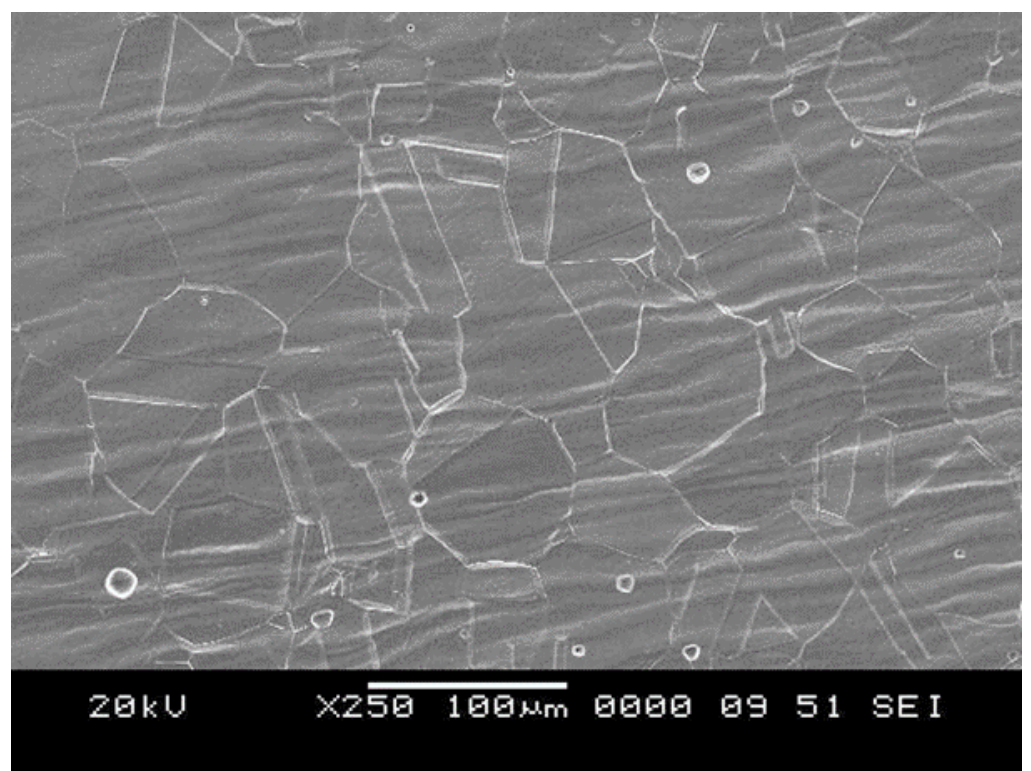

Figure 1. Microstructure of hopper pipe showing austenite grains

\section{RESULTS}

\section{Visual Inspection}

Figure 2a shows the dismantled failed hopper pipe at the discharge unit, that failed during the service. Further, the pipe was removed from the discharge unit and visual examination was carried out on the failed hopper pipe in order to have the better understanding of the failure. Figure $2 \mathrm{~b}$ and $2 \mathrm{c}$ show the close-up images of the fractured pipe and its location. Visual inspection confirms that the pipe suffered a transverse fracture leading to failure of hopper discharge unit. It is to be noted that the fracture region is close to the welded region. Also, corrosion products were observed in the fractured region (Figure 2d), indicating the presence of aggressive environment around the hopper pipe. 

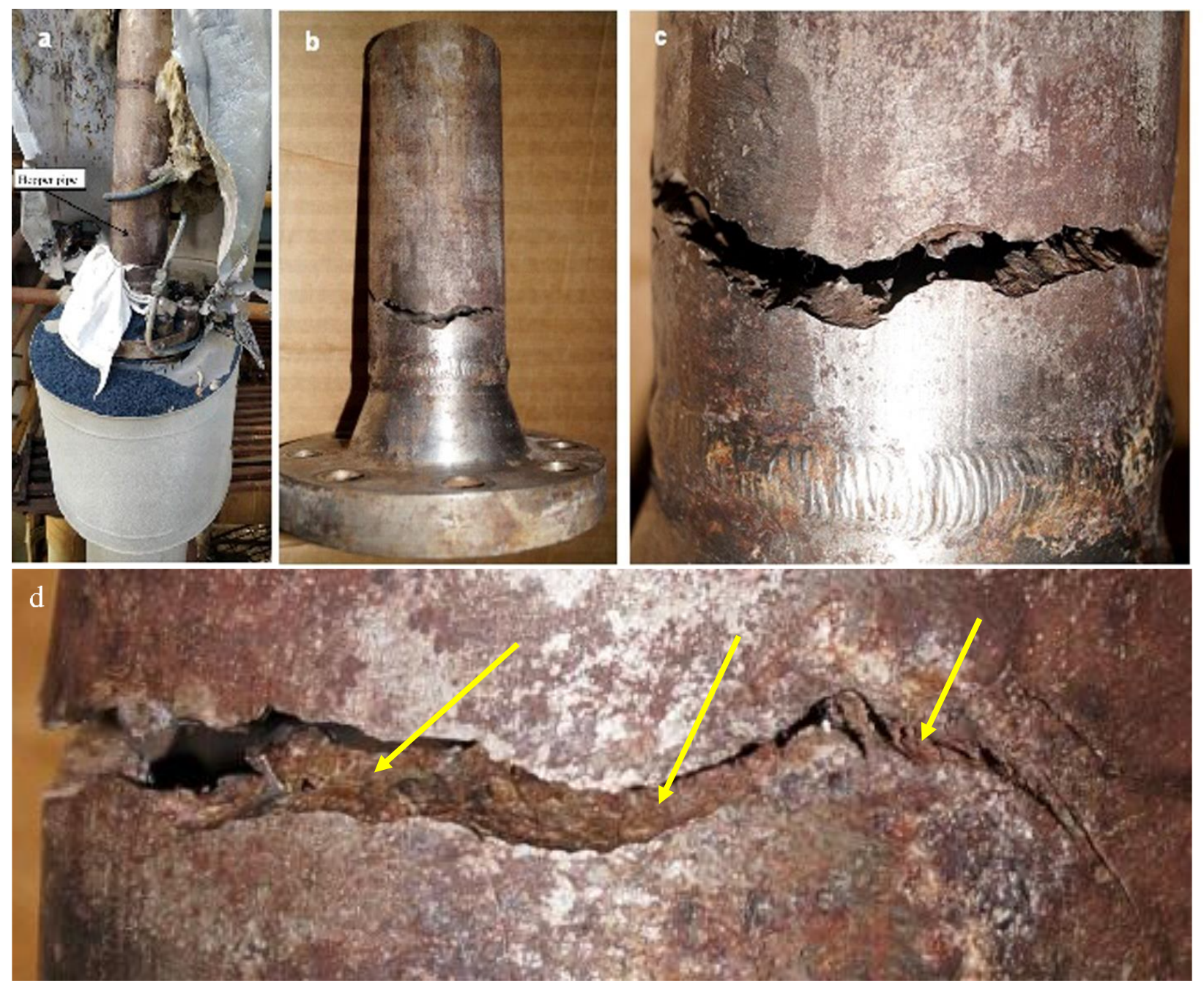

Figure 2. Visual inspection images showing, (a) failed industrial discharge hopper pipe (b) fractured pipe (c) close up view at fracture location (d) corrosion products observed in the fracture location

\section{Non Destructive Testing}

Dye Pentrant Inspection (DPI) test was carried out on the fractured hopper pipe to understand the crack initiation and crack propogation. As shown in Figure 3, many minor cracks were observed on the outer surface of the pipe along with main prominant transverse fracture that caused the failure of hopper pipe. It is to be noted that these minor cracks are not oriented in a particular direction and instead they are orienting in random directions. 


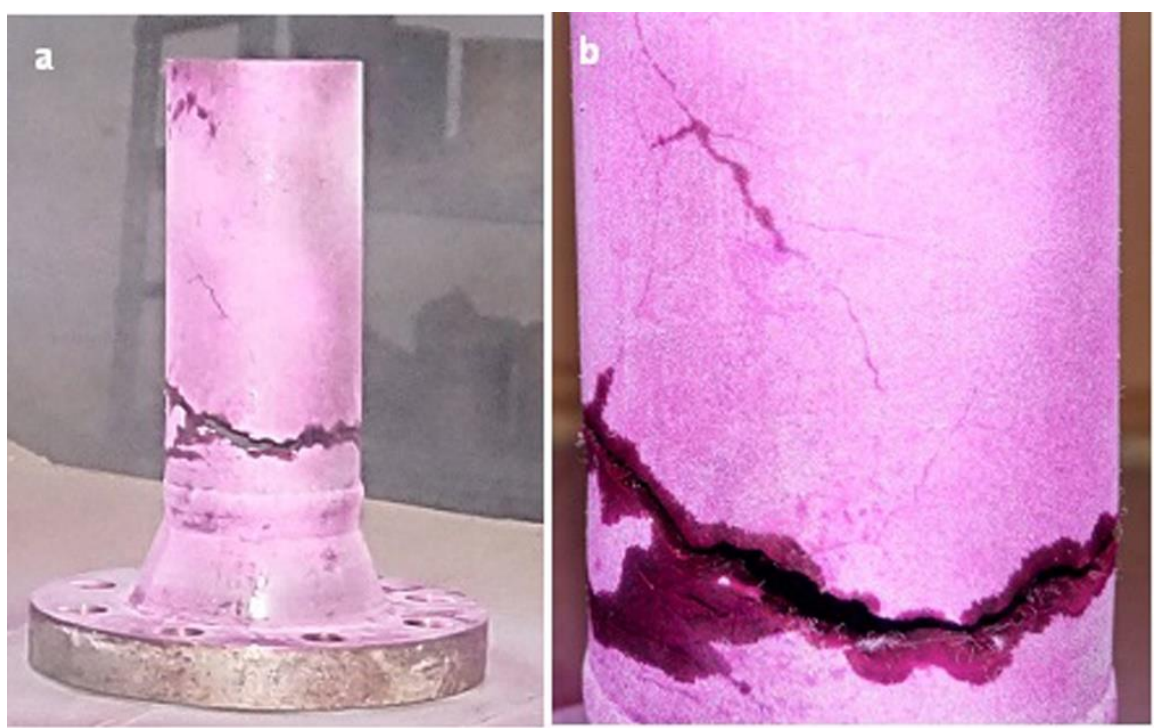

Figure 3. Dye Penetrant Inspection Test images showing, (a) crack propogation (b) close up view of fracture location

\section{Metallography of the Fracture}

A section having a crack on the surface was sectioned from the hopper pipe and was subjected to detailed metallographic study. Standard metallurgical procedure involving belt grinding, polishing with emery papers, was followed to prepare the specimen for metallurgical analysis. Further, to obtain mirror finish the specimen was polished in a disc polishing unit with 1 micron alumina followed by 0.5 micron diamond polisher. Unetched specimen, observed under the optical microscope revealed crack branches oriented in different directions as shown in Figure 4a. Specimen was then etched to observe how crack pattern is associated with microstructure. Figure $4 \mathrm{~b}$ shows crack propagation indicating transgranular fracture. Further, the specimen was observed under SEM to confirm the transgranular nature of fracture. SEM images as shown in Figure 5, clearly showed crack propagation cutting through the austenite grains, confirming the transgranular nature of fracture. Energy dispersion spectrometer in combination with SEM was used to analyze the chemical composition of the corroded zone observed in the transgranular fracture. As shown in Figure 6, Chlorine content was clearly observed, along with other major elements of SS 316, indicating chloride formation which might have led to stress corrosion cracking.
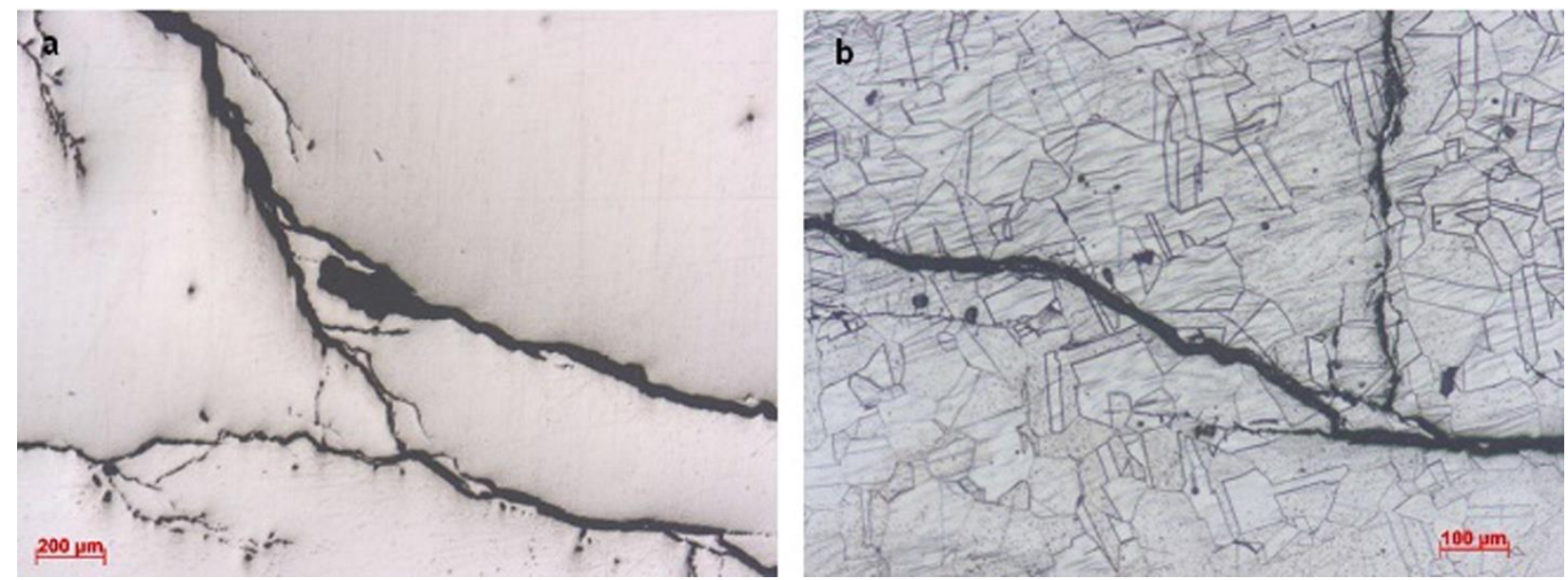

Figure 4. Optical images showing transgranular fracture: (a) in unetched condition (b) etched condition 

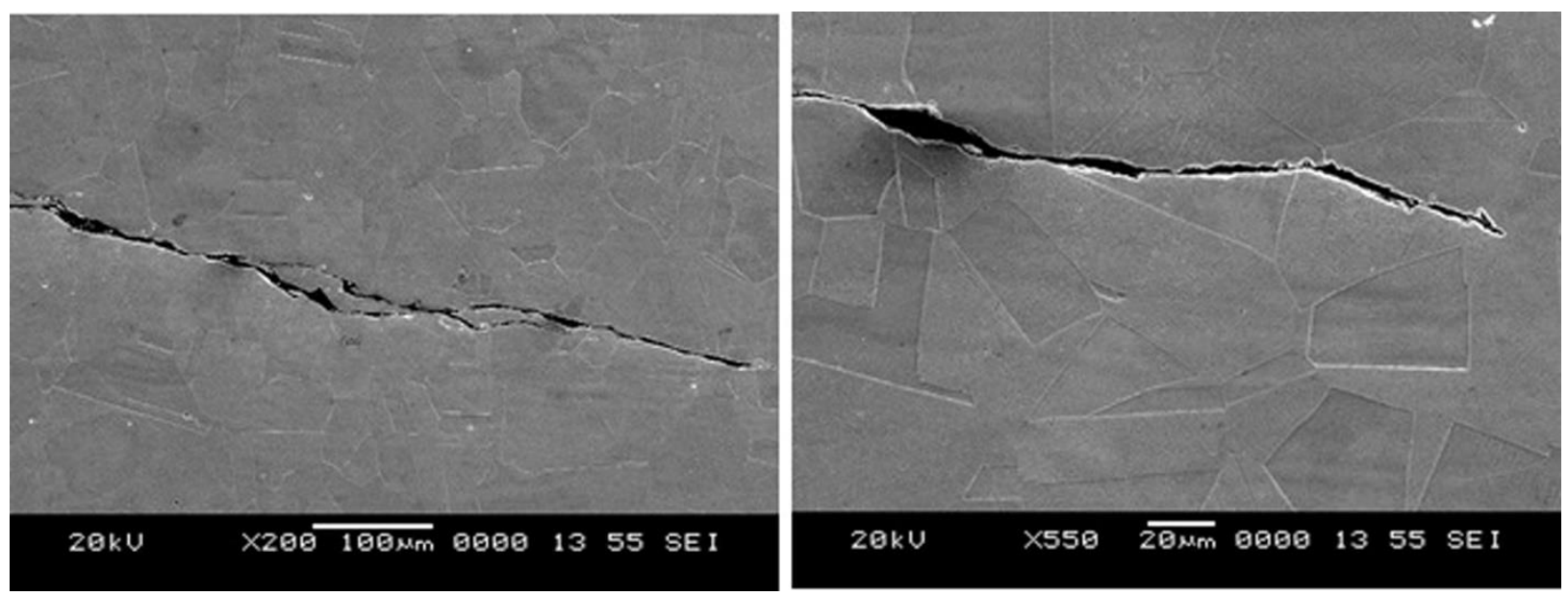

Figure 5. SEM images showing transgranular fracture
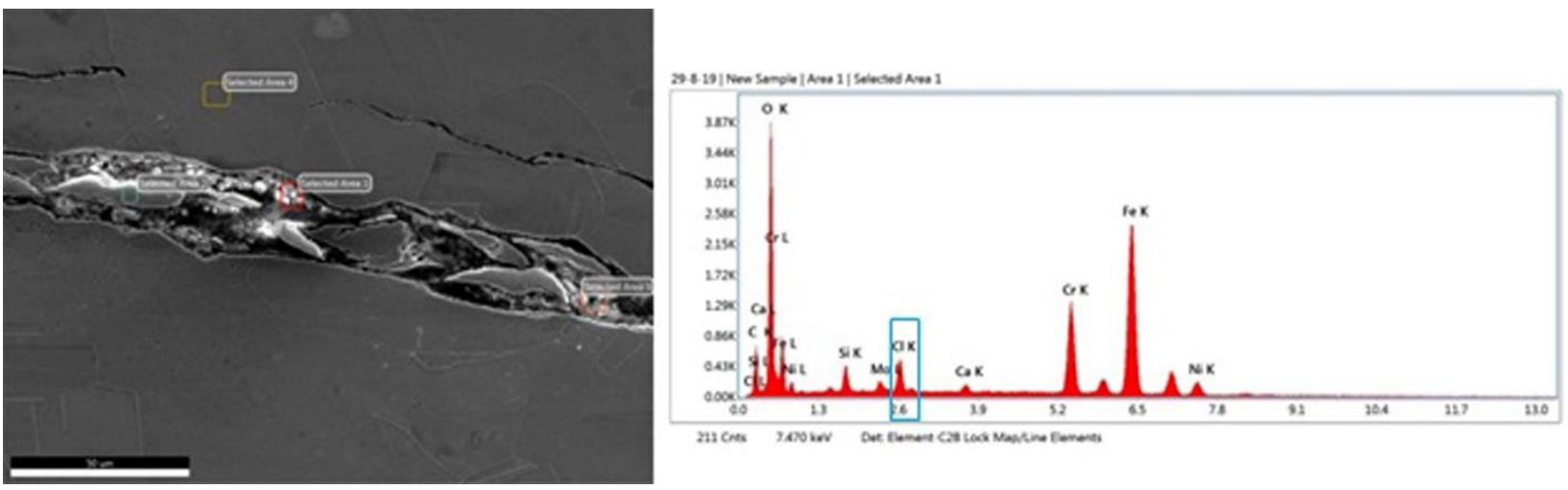

Figure 6. EDX showing chlorine content in the fractured region

\section{Fratography}

One of the minor cracks was opened and was observed under the SEM to study the mode of fracture. Portion of the pipe having a small crack was sectioned using power hacksaw, leaving around $2 \mathrm{~cm}$ each from both the sides of the crack. Care was taken to protect the fracture surface as it was covered with a Teflon tape to avoid any surface debris or water, flowing through it. Once the specimen containing fracture was obtained, it was held in the grippers of tensile testing machine with crack at the center. Using this setup, uniform load was applied and the specimen was separated into two parts without damaging the fracture surface. In general, transgranular nature of the fracture is asssociated with either cleavage or striations [7]. In this case, SEM images observed at the fractured region showed striations within the grains, indicating fatigue loading condition as shown in Figure 7.
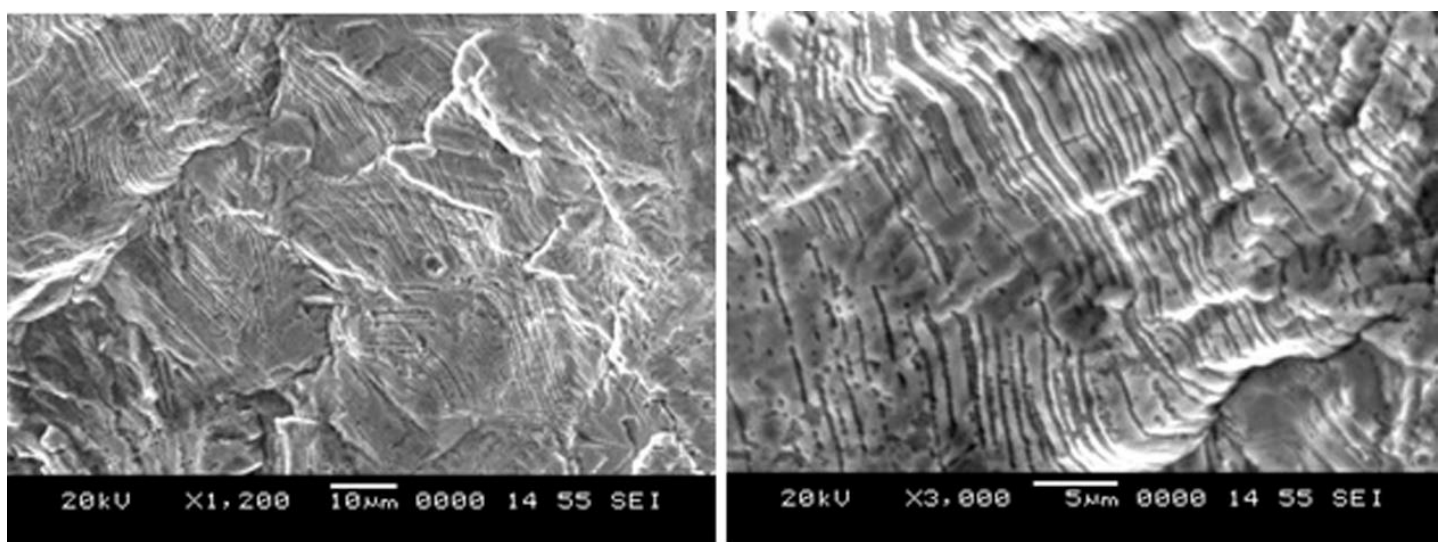

Figure 7. Fractograph images showing striations within the grains 


\section{DISCUSSION}

Although SS 316 grade posseses corrosion resistance properties, visual inspection of the fractured hopper pipe clearly showed transverse fracture near the weld region and corrosion products were also observed within the fractured region. Further, Dye Penetration Inspection highlighted minor fractures orienting at different directions along with the major transverse fracture, indicating possibility of more cracks. This indicates that the pipe has been subjected to corrosion because of service conditions/environment and any of those minor cracks would have opened up with the continued service operation, had the major transverse fracture was not taken place. It also indicates that the major crack could be the result of joining of minor cracks or propagation of a single major crack with a prolonged service operation. Critical observations made through metallographic study showed that, the crack is propagating in a transgranular fashion, cutting the austenite grains of SS 316. It is to be noted that the possibility of sensitization that typically occurs in 300 series of stainless steels, has to be ruled out as the operating temperature of the hopper pipe $\left(140{ }^{\circ} \mathrm{C}\right)$ was much lower than the sensitization range $\left(480\right.$ to $\left.815^{\circ} \mathrm{C}\right)$ [7]. Transgranular fracture in a stainless steel is a typical feature of stress corrosion cracking and to support that, EDX analysis carried out at the corrosion products, formed at the fracture surface showed presence of Chlorine content. Presene of chlorine content on the outer surface of hopper pipe, in combination with oxygen present in the working condition formed chloride and acted as the main source for SCC. Fractography carried out on the fracture surface showed striations, indicating that the material was under fatigue loading condition. Hence, the stresses present in the pipe because of fatigue loading, in combination with chloride formation at the surface, led to stress corrosion cracking, leading to failure of hopper pipe.

\section{CONCLUSION}

The detailed investigation carried out on fractured hopper pipe of an oil and natural gas industry concludes that, stress corrosion cracking is the root cause of failure. Chloride formation at the outer surface degraded the material and acted as the crack initiation point. Further, the fatigue stresses because of service conditions present in the pipe accentuated the propagation of crack in a transgranular fashion leading to stress corrosion cracking. Cathodic protection or suitable polymer coating should be considered as the preventive measures to avoid stress corrosion craking in future.

\section{ACKNOWLEDGEMENT}

Authors thank the Department of Metallurgical and Materials Engineering, National Institute of Technology Karnataka, for providing experimental facilities for completing this work.

\section{REFERENCES}

[1] R. SONG, J. XIANG, and D. HOU, "Characteristics of Mechanical Properties and Microstructure for 316L Austenitic Stainless Steel,” J. Iron Steel Res. Int., vol. 18, no. 11, pp. 53-59, 2011.

[2] M. A. M. Ibrahim, S. S. Abd El Rehim, and M. M. Hamza, "Corrosion behavior of some austenitic stainless steels in chloride environments," Mater. Chem. Phys., vol. 115, no. 1, pp. 80-85, 2009.

[3] J. Swaminathan, R. Singh, M. K. Gunjan, and B. Mahato, "Sensitization induced stress corrosion failure of AISI 347 stainless steel fractionator furnace tubes," Eng. Fail. Anal., vol. 18, no. 8, pp. 2211-2221, 2011.

[4] M. Suresh Kumar, M. Sujata, M. A. Venkataswamy, and S. K. Bhaumik, "Failure analysis of a stainless steel pipeline," Eng. Fail. Anal., vol. 15, no. 5, pp. 497-504, 2008.

[5] C. Barbosa, S. M. C. de Souza, R. O. Centeno, I. C. Abud, and O. B. Ferraz, "Failure analysis of pipes used in a hydrodesulfuration system of a petrochemical plant,” Eng. Fail. Anal., vol. 13, no. 7, pp. 1076-1091, 2006.

[6] T. N. Prasanthi et al., "Failure analysis of a 304 steel component aged at 623K," Eng. Fail. Anal., vol. 31, pp. 28-39, 2013.

[7] Asm, ASM Handbook, no. v. 12. ASM International, 1986.

[8] E. Sadeghi Meresht, T. Shahrabi Farahani, and J. Neshati, "Failure analysis of stress corrosion cracking occurred in a gas transmission steel pipeline," Eng. Fail. Anal., vol. 18, no. 3, pp. 963-970, 2011.

[9] C. Manfredi and J. L. Otegui, "Failures by SCC in buried pipelines,” Eng. Fail. Anal., vol. 9, no. 5, pp. 495-509, 2002.

[10] F. Fantechi and M. Innocenti, "Chloride stress corrosion cracking of precipitation hardening S.S. impellers in centrifugal compressor. Laboratory investigations and corrective actions," Eng. Fail. Anal., vol. 8, no. 5, pp. 477-492, 2001.

[11] G. Yang, K. B. Yoon, and Y. C. Moon, "Stress corrosion cracking of stainless steel pipes for Methyl-Methacrylate process plants," Eng. Fail. Anal., vol. 29, pp. 45-55, 2013.

[12] S. M. R. Ziaei, J. Mostowfi, M. Golestani pour, and S. A. R. Ziaei, "Failure analysis: Chloride stress corrosion cracking of AISI 316 stainless steel downhole pressure memory gauge cover," Eng. Fail. Anal., vol. 33, pp. 465-472, 2013.

[13] N. Li et al., "Stress Corrosion Cracking of an Electrohydraulic Oil Pipe," J. Fail. Anal. Prev., vol. 19, no. 1, pp. 29-35, 2019.

[14] T. G. Kim, "Failure of piping by hydrogen attack," Eng. Fail. Anal., vol. 9, no. 5, pp. 571-578, 2002. 